\title{
Exclusive Meson Production at NLO
}

\author{
Markus Diehl and Wolfgang Kugler \\ Deutsches Elektronen-Synchroton DESY \\ 22603 Hamburg, Germany
}

\begin{abstract}
We report on numerical studies of the NLO corrections to exclusive meson electroproduction, both in collider and fixed-target kinematics. Corrections are found to be huge at small $x_{B}$ and sizeable at intermediate or large $x_{B}$.
\end{abstract}

\section{Motivation and general framework}

Generalized parton distributions (GPDs) are a versatile tool to quantify important aspects of hadron structure in QCD. They contain unique information on the spatial distribution of partons [1] and on the orbital angular momentum they carry in the proton [2]. The theoretically cleanest process where GPDs can be studied is deeply virtual Compton scattering (similar to inclusive DIS, which plays a dominant role in constraining the usual parton densities). Hard exclusive meson production is harder to describe quantitatively, but it provides opportunities to obtain important complementary constraints. In particular, vector meson production is more directly sensitive to the gluon distributions, which enter the Compton amplitude only at next-to-leading (NLO) order in $\alpha_{s}$. Together with a wealth of high-quality data [3], this warrants efforts to bring meson production under theoretical control as much as possible.

In the present contribution [4] we investigate exclusive $\rho$ production $\left(\gamma^{*} p \rightarrow \rho p\right)$ using collinear factorization, which is applicable in the limit of large photon virtuality $Q^{2}$ at fixed Bjorken variable $x_{B}$ and fixed invariant momentum transfer $t$ to the proton [5]. In practical terms, this means that the description is restricted to sufficiently large $Q^{2}$ but can be used for both small and large $x_{B}$, thus providing a common framework for analyzing both collider and fixed-target data. The process amplitude can then be expressed in terms of GPDs for the proton, the $q \bar{q}$ distribution amplitude for the $\rho$, and hard-scattering kernels. The kernels are known to NLO, i.e. to order $\alpha_{s}^{2}[6]$.

The requirement of "sufficiently large" $Q^{2}$ is demanding for meson production. Contributions that are formally suppressed by powers of $1 / Q^{2}$ cannot be calculated in a completely systematic way, but the estimates $[7,8,9]$ agree that for $Q^{2}$ of several $\mathrm{GeV}^{2}$ the effect of the transverse quark momentum inside the meson cannot be neglected in the hard-scattering subprocess, as it is done in the collinear approximation. This effect can be incorporated in the modified hard-scattering picture $[7,8]$, in color dipole models [9], or in the MRT approach [10]. Unfortunately, the calculation of $\alpha_{s}$ corrections remains not only a technical but even a conceptual challenge in these approaches, so that the perturbative stability of their results cannot be investigated at present. One strategy in this situation is to study the NLO corrections in the collinear factorization framework, identifying kinematical regions where they are moderate or small. There one may use formulations incorporating power corrections from transverse quark momentum with greater confidence. This is the aim of the present contribution.

In the following we show results for the convolution of the unpolarized quark and gluon GPDs $H^{q}$ and $H^{g}$ with the corresponding hard-scattering kernels and the asymptotic form of the $\rho$ distribution amplitude. We model the GPDs using a standard ansatz based on double 

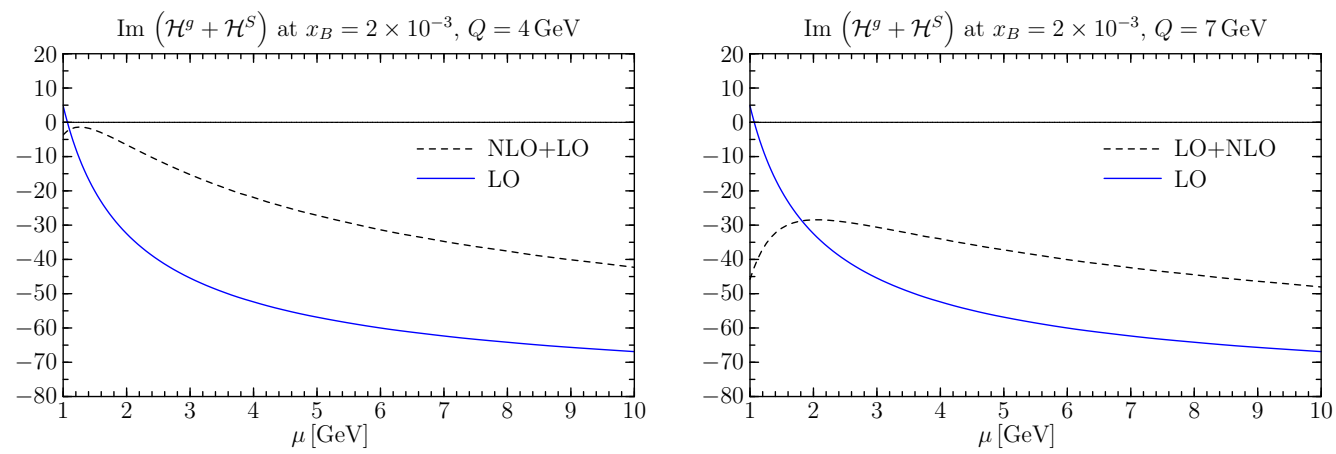

Figure 1: Imaginary part of the convolution integral for the sum of gluon and quark singlet distributions as a function of the renormalization and factorization scale $\mu$.

distributions [11], with the CTEQ6M distributions as input. Unless indicated explicitly, we take $t=0$ and set the factorization and renormalization scales equal, $\mu=\mu_{F}=\mu_{R}$.

\section{Numerical results}

In a wide kinematical range at small $x_{B}$, we find huge NLO corrections which have opposite sign to the Born term and almost cancel it. This is shown for $x_{B}=2 \times 10^{-3}$ in Fig. 1, where there are indications for an onset of perturbative stability at $Q=$ $7 \mathrm{GeV}$, but not yet at $Q=4 \mathrm{GeV}$. Taking $x_{B}=2 \times 10^{-4}$ one finds no stability even at $Q=7 \mathrm{GeV}$, whereas for $x_{B}=2 \times 10^{-2}$ the corrections are of tolerable size already at $Q=4 \mathrm{GeV}$.

Figure 2 shows that in kinematics relevant for HERA measurements, NLO corrections have a huge effect on the cross section and moreover lead to a flat energy behavior in conflict with experiment. Due to the strong cancellations between LO and NLO terms, the dependence on factorization and renormalization scale is not reduced when going to NLO.

As already observed in [6] the large size

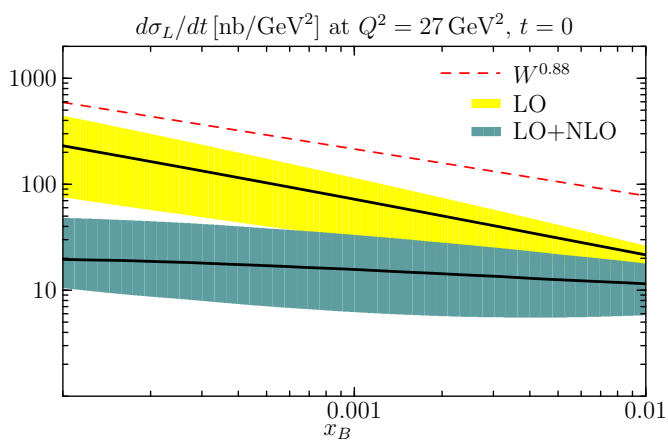

Figure 2: Cross section for $\gamma^{*} p \rightarrow \rho p$ with a longitudinal photon. Bands correspond to the range $Q / 2<\mu<2 Q$ and solid lines to $\mu=Q$. We also show the power-law behavior $\sigma \propto W^{0.88}$ (with arbitrary normalization) obtained from a fit to data in the range $0.001 \lesssim x_{B} \lesssim 0.005$ [12]. of NLO corrections at small $x_{B}$ can be traced back to BFKL-type logarithms appearing first at NLO for vector meson production. Such logarithms are present in many processes (including DIS) but have a rather large numerical prefactor in the present case. It is to be hoped that all-order resummation of 

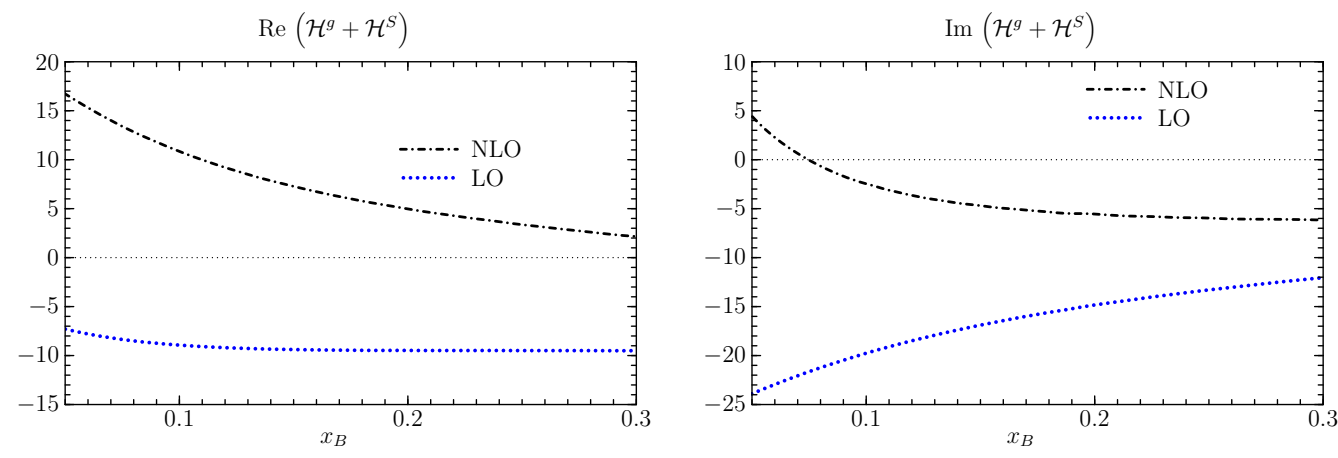

Figure 3: Real and imaginary part of the convolution integral for the sum of gluon and quark singlet distributions for $\mu_{F}=\mu_{R}=Q=2 \mathrm{GeV}$.
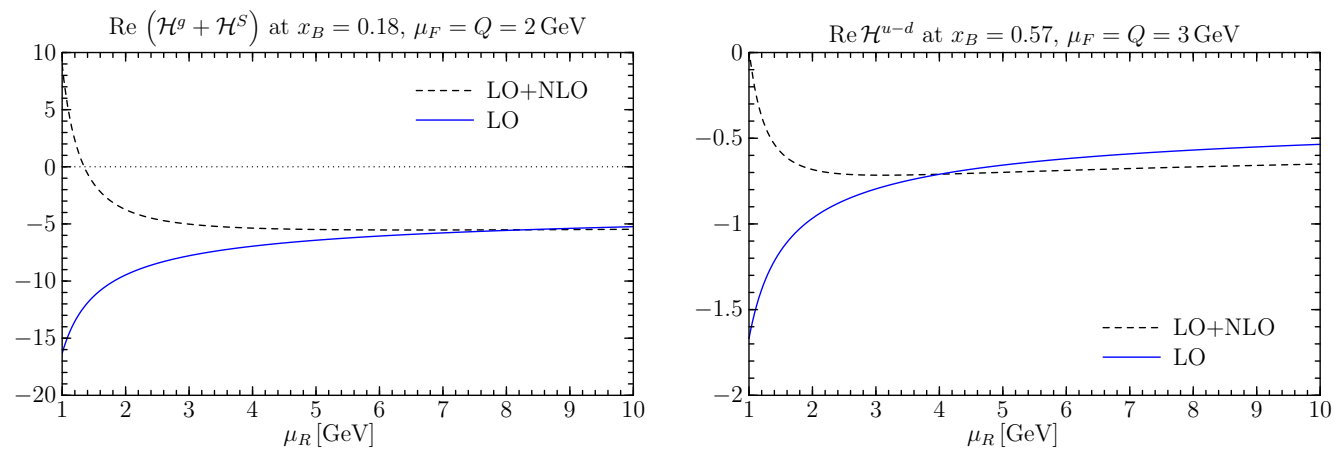

Figure 4: Renormalization scale dependence of the real part of the convolution integrals for the sum of gluon and quark singlet distributions (left) and for for the difference of $u$ and $d$ quark distributions (right).

these logarithms in the hard-scattering kernel will give perturbative stability at small $x_{B}$.

In the $x_{B}$ range relevant for experiments at COMPASS, HERMES, and JLAB, we generally find corrections which are sizable but not huge. An exception is the real part of the gluon and quark singlet amplitudes, where corrections become large for decreasing $x_{B}$, as is seen in the left panel of Fig. 3.

In the quark nonsinglet sector there are large terms in the NLO kernel due to gluon self-energy corrections. The BLM procedure for setting the renormalization scale aims at resumming these to all orders in $\alpha_{s}$. Applied to the process at hand, one finds however that this requires $\mu_{R}$ to be typically an order of magnitude smaller than $Q[13,14]$. This is outside the validity of the perturbative calculation for most practically relevant $Q$. Numerically we find that for $\mu_{R} \lesssim 2 \mathrm{GeV}$ the NLO corrections become unstable for several convolution integrals, as shown for examples in Fig. 4.

We have therefore omitted this region when estimating the scale setting error in Fig. 5, where we show the cross section in typical fixed-target kinematics. We see that NLO correc- 

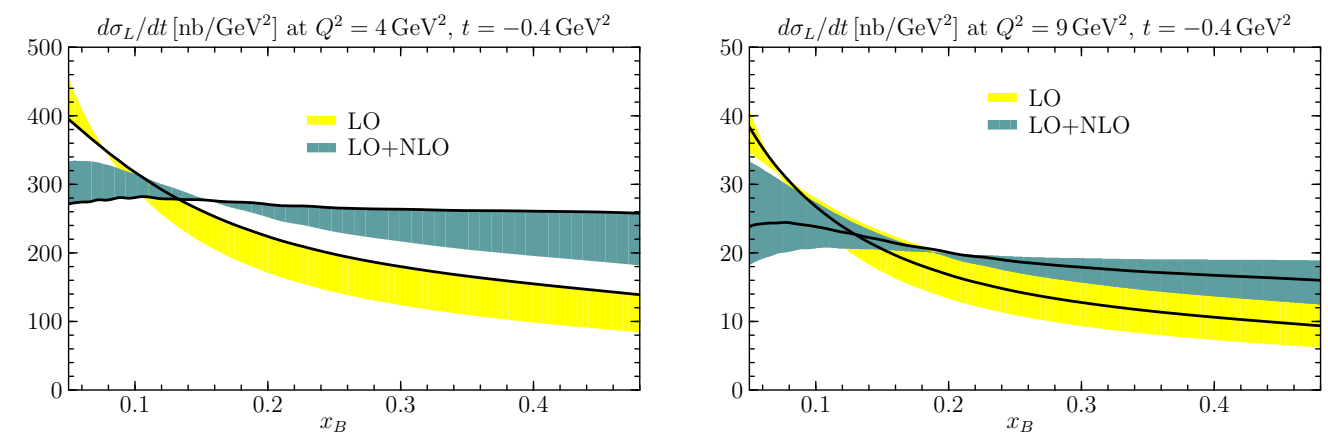

Figure 5: Cross section for $\gamma^{*} p \rightarrow \rho p$ with a longitudinal photon. Bands correspond to the range $2 \mathrm{GeV}<\mu<4 \mathrm{GeV}$ in the left and to $2 \mathrm{GeV}<\mu<6 \mathrm{GeV}$ in the right plot, and solid lines to $\mu=Q$ in both cases.

tions are quite large for $Q^{2}=4 \mathrm{GeV}^{2}$, whereas for $Q^{2}=9 \mathrm{GeV}^{2}$ and $x_{B}>0.1$ they become moderate.

\section{Acknowledgments}

We gratefully acknowledge discussions with L. Favart, H. Fischer, D. Yu. Ivanov, A. Rostomyan and A. Schäfer. This work is supported by the Helmholtz Association, contract number VH-NG-004.

\section{References}

[1] M. Burkardt, Int. J. Mod. Phys. A 18 (2003) 173 [hep-ph/0207047].

[2] X. D. Ji, Phys. Rev. Lett. 78 (1997) 610 [hep-ph/9603249].

[3] A. Levy, these proceedings.

[4] Slides: http://indico.cern.ch/contributionDisplay $\cdot$ py? contribId=83\&sessionId=12\&conf Id=9499

[5] J. C. Collins, L. Frankfurt and M. Strikman, Phys. Rev. D 56 (1997) 2982 [hep-ph/9611433].

[6] D. Yu. Ivanov, L. Szymanowski and G. Krasnikov, JETP Lett. 80 (2004) 226 [hep-ph/0407207].

[7] S. V. Goloskokov and P. Kroll, Eur. Phys. J. C 50 (2007) 829 [hep-ph/0611290]; P. Kroll, these proceedings, arXiv:0706.2587 [hep-ph].

[8] M. Vanderhaeghen, P. A. M. Guichon and M. Guidal, Phys. Rev. D 60 (1999) 094017 [hep-ph/9905372].

[9] L. Frankfurt, W. Koepf and M. Strikman, Phys. Rev. D 54 (1996) 3194 [hep-ph/9509311].

[10] A. D. Martin, M. G. Ryskin and T. Teubner, Phys. Rev. D 55 (1997) 4329 [hep-ph/9609448]; T. Teubner, these proceedings.

[11] I. V. Musatov and A. V. Radyushkin, Phys. Rev. D61 (2000) 074027 [hep-ph/9905376].

[12] ZEUS Collaboration, paper 594 submitted to EPS 2001, Budapest, Hungary, http://www-zeus.desy.de/physics/phch/conf/eps01_paper.html

[13] A. V. Belitsky and D. Müller, Phys. Lett. B 513 (2001) 349 [hep-ph/0105046].

[14] I. V. Anikin et al., Eur. Phys. J. C 42 (2005) 163 [hep-ph/0411408]; S. J. Brodsky and F. J. Llanes-Estrada, Eur. Phys. J. C 46 (2006) 751 [hep-ph/0512247]. 